\title{
Adaptation of microbial communities in soil contaminated with polychlorinated biphenyls, leading to the transformation of more highly chlorinated congeners in biofilm communities
}

\footnotetext{
* Corresponding author: Dr W.-R. Abraham

Helmholtz Center for Infection

Research

Chemical Microbiology

Inhoffenstrasse 7

38124 Braunschweig

Germany

T 4953161814300 ,

F 4953161814699 ,

Ewab@gbf.de

1 Helmholtz Center for Infection Research (former GBF), Chemical Microbiology, Inhoffenstrasse 7, 38124 Braunschweig, Germany

2 Helmholtz Centre for Environmental Research - UFZ, Department of River Ecology, Brückstrasse 3a, 39114 Magdeburg, Germany

Date submitted: 10.01 .07

Date accepted: 11.06 .07
}

\author{
A. J. Macedo', T. R. Neu², U. Kuhlicke ${ }^{2}$ and \\ W.-R. Abraham ${ }^{1 *}$
}

\begin{abstract}
A site polluted for many years with polychlorinated biphenyls (PCB) was used to elucidate the metabolic adaptation of microbial communities to these xenobiotics. Soil samples taken along a gradient of PCB-pollution at this site were used to grow biofilm communities on PCB oil. The biofilm communities originating from the non-polluted soil formed rather uniform and thin bacterial layers on PCB oil, while the biofilms originating from contaminated soil samples formed agglomerated structures on the PCB droplets. Biofilm communities were very diverse but those from highly polluted soil were dominated by Burkholderia species, a genus known for degrading several PCBs. All biofilm communities could transform low to medium chlorinated PCB congeners but a strong increase in the rate and degree of PCB transformation in communities from heavily polluted soil was observed. Notably, pentachlorinated congeners were transformed only by biofilms derived from the highly polluted soil but at the same time the content of trichlorinated congeners did not decrease. It is assumed that biofilms from the highly contaminated soil reductively dechlorinated PCB, converting pentachlorinated congeners to trichlorinated congeners in the spherical biofilm aggregates by diffusing to the surface of the aggregates, where aerobic transformation took place.
\end{abstract}

\section{INTRODUCTION}

A broad spectrum of organic compounds has been produced by humankind that were new to microbes already present in the environment. Polychlorinated biphenyls (PCB) are compounds of this type, consisting of a biphenyl core substituted by chlorines, permitting the formation of up to 209 different compounds, called congeners. Owing to their high chemical and thermal stability they became very popular in the 1970s for industrial applications (Abraham et al., 2002). The first report of PCB in environmental samples dated from the late 1960s (Jensen et al., 1969) and nowadays it is estimated that a considerable fraction of the $1.5 \times 10^{6}$ tonnes produced worldwide entered the environment. The high hydrophobicity of PCB causes a strong accumulation of these xenobiotics in fat. Food consumption contributes to more than $90 \%$ of the human PCB exposure, owing to enrichments along the food chains. Evidence indicates that
PCB cause a diversity of health effects including cancer and affect the immune, reproductive, nervous and endocrine systems (Ross, 2004).

How do bacteria react to the sudden exposure to the xenobiotic PCB, which are scarcely bioavailable because of their hydrophobicity and which are unknown to bacterial metabolism because of a lack of similar structures occurring naturally? We know that some bacteria, at least, can handle these compounds, as a number of strains that can use lower chlorinated PCB congeners as substrates have been isolated from polluted sites. However, in situ bioremediations of PCB-contaminated sites are difficult, even using optimized strains (Harkness et al., 1993). To understand the behaviour of autochthonous microbial communities in exposures to such hydrophobic substrates we studied a PCB-contaminated site where leaking PCB from electrical transformers contaminated the soil in such high concentrations that, in some places, PCB became the main carbon source (Nogales et al., 1999). The 
microbial communities at this site as well their activity were characterized in several studies (Nogales et al., 1999, 2001; Lünsdorf et al., 2000). Recently, we described a complex process of biofilm maturation on PCB oil using a soil sample from this PCB-contaminated site (Macedo et al., 2005) and we address here the question how such biofilm communities may vary between soil samples of different PCB contents. The approach of our study was to expose PCB oil droplets to microbial communities from soil samples taken along a PCB-pollution gradient and to monitor the formation and performance of biofilm communities on the PCB oil. The rationale was that biofilm microbiology has become a very important area of research since the realization that biofilms are widely distributed in the environment and in diseases (HallStoodley et al., 2004). Microorganisms organized in biofilms are less prone to environmental stress (Schachter, 2003; Walker et al., 2003) and develop a high level of worksharing including establishment of metabolic networks (Tillmann et al., 2005). Because of the resistance of biofilm communities to hostile conditions we speculated that biofilms should be the optimal lifestyle to handle the hydrophobicity and toxicity of PCB. Biofilms developing on the PCB oil were analysed using a polyphasic approach that combined community fingerprinting with $16 \mathrm{~S}$ rRNA gene sequence analyses, chemical analysis of PCB transformation and confocal laser scanning microscopy (CLSM) to characterize the biofilm structure. From several techniques for community fingerprinting, single-strand conformation polymorphism (SSCP) was chosen, which permitted the analysis of amplicons of up to 371 basepairs. We report here on the diversity and the performance of PCB transformation of biofilm microbial communities along a pollution gradient in soil samples.

\section{MATERIAL AND METHODS}

\section{Soil samples}

The site situated north of Wittenberg, Germany $\left(51^{\circ}\right.$ $54.920^{\prime} \mathrm{N} / 12^{\circ} 43.785^{\prime} \mathrm{E}$ ), had been polluted with $\mathrm{PCB}$ oil more than a decade ago. Three different soil samples (300$500 \mathrm{~g}$ ) were taken along a PCB-pollution gradient (Table 1 and Fig. 1a) and stored at $4{ }^{\circ} \mathrm{C}$ until use within 2 days. Soil sample SW-NC1 had less than $0.2 \mathrm{mg} \mathrm{PCB} / \mathrm{kg}$ soil (which was the detection limit for our PCB analysis), soil sample SW-027 $27 \mathrm{mg}$ PCB/kg soil and soil sample SW-813 813 $\mathrm{mg} \mathrm{PCB} / \mathrm{kg}$ soil. Homogenized, freeze-dried soil samples (a)

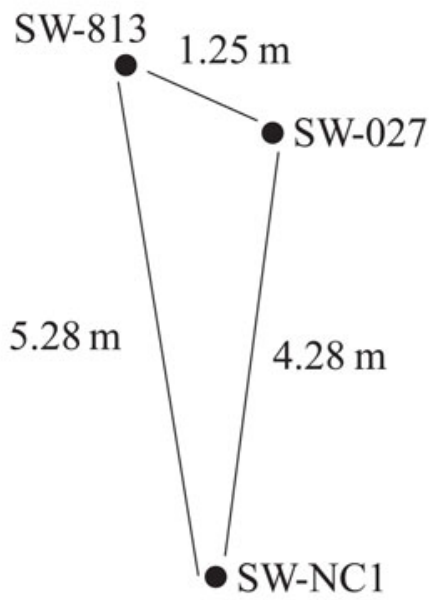

(b)

Floating slide carrying PCB droplets

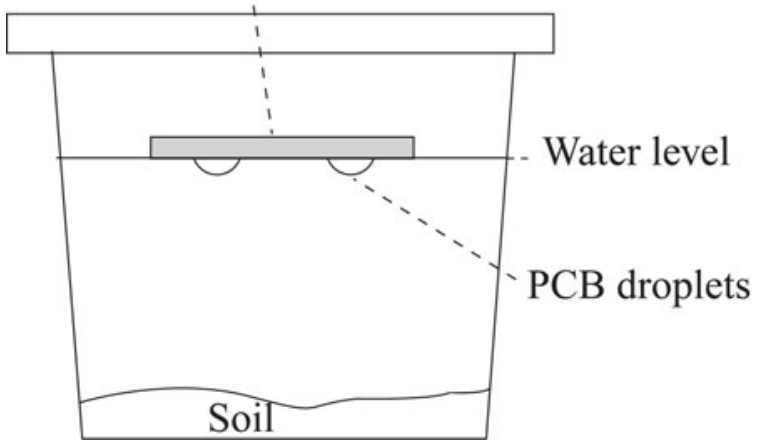

Fig. 1: (a) Sampling sites and (b) scheme of the microcosm used for the biofilm formation.

(1 mg) were combusted in a Fisons EA 1108 element analyser with $\mathrm{CHN}$ packing for analysis of total organic carbon (TOC) and total nitrogen (TN). The analyses were run five times (Macedo et al., 2005). The soil samples consisted mainly of sand with some clay, were acidic $(\mathrm{pH}$ 4.47-4.90) and had low TOC and TN (Table 1). The PCB mixture was found to consist mostly of low-chlorinated congeners with small amounts of pentachloro-PCB: $2.9 \%$

Table 1: Soil characteristics of soil samples used as inocula in the microcosm experiments

\begin{tabular}{lcclll}
\hline Soil sample & $\mathrm{pH}$ & $\begin{array}{l}\text { PCB content } \\
(\mathrm{mg} \text { PCB/kg soil })\end{array}$ & $\begin{array}{l}\text { Total organic carbon } \\
(\mathrm{g} \text { TOC/kg soil })\end{array}$ & $\begin{array}{l}\text { Total nitrogen } \\
\text { (g TN/kg soil) }\end{array}$ & C:N ratio \\
\hline SW-NC1 & 4.90 & $0 \pm 0.4^{a}$ & $1.4 \pm 0.4^{b}$ & $0.1 \pm 0.03$ & 14.0 \\
SW-027 & 4.47 & $27 \pm 11$ & $7.4 \pm 1.9$ & $0.4 \pm 0.10$ & 18.5 \\
SW-813 & 4.54 & $813 \pm 24$ & $3.8 \pm 0.7$ & $0.2 \pm 0.04$ & 19.0 \\
\hline
\end{tabular}

PCB, polychlorinated biphenyls; TOC, total organic carbon; TN, total nitrogen.

${ }^{a}$ Detection limit was $0.4 \mathrm{mg} \mathrm{PCB} / \mathrm{kg}$ soil; standard deviations were based on six measurements.

${ }^{b}$ Standard deviations were based on five measurements. 
dichloro-, $44.7 \%$ trichloro-, $51.6 \%$ tetrachloro-, and $0.8 \%$ pentachloro-biphenyls (Nogales et al., 1999). Such a mixture of PCB congeners is very similar to Aroclor 1242; therefore this PCB mixture was used in our microcosm experiments (Ivanov \& Sandell, 1992).

\section{PCB analysis}

PCBs were extracted using a modified procedure (Macedo et al., 2005) as described by Buthe \& Denker (1995). Briefly, three pieces of Permanox slide (eight for the control without inoculation with the soil sample) for each microcosm, each bearing one PCB droplet, were cut off the slides and were separately extracted with $n$ hexane, the extracts were cooled to $0{ }^{\circ} \mathrm{C}$, and concentrated under a gentle stream of $\mathrm{N}_{2}$, the residues were dissolved in $1 \mathrm{ml}$ hexane. Capillary gas chromatographic analyses were performed on $1 \mu \mathrm{l}$ aliquots of the extract on a Hewlett Packard 5890 Series II gas chromatograph equipped with a HP Ultra 2 capillary column $(50 \mathrm{~m}$ by $0.2 \mathrm{~mm}$; film thickness $0.11 \mathrm{~mm}$ ) and flame ionization detector. Hydrogen served as the carrier gas. The injector temperature was set to $250{ }^{\circ} \mathrm{C}$ and the detector temperature to $300^{\circ} \mathrm{C}$. The oven program was: $80^{\circ} \mathrm{C}$ for $3 \mathrm{~min}, 80^{\circ} \mathrm{C}$ to $288^{\circ} \mathrm{C}$ at $6 \mathrm{deg} . \mathrm{C} / \mathrm{min}$ followed by an isothermal period of $20 \mathrm{~min}$. The PCB congeners were identified by gas chromatographymass spectrometry (GC-MS) and by comparison with authenticated standards. The 2,2',5,5'-tetrachloro-PCB (PCB 52) (Ballschmiter \& Zell, 1980) present in Aroclor 1242 is assumed not to be leached by water (Ayris \& Harrad, 1999) and not to be aerobically transformable (Duinker et al., 1988); therefore it served as internal standard (Duinker et al., 1988). The chromatograms were normalized to the area of PCB 52 of Aroclor 1242. The GCMS analyses were performed with a gas chromatograph similar to that described (same column and conditions but helium as carrier gas) connected to an HP 5989A quadrupole mass spectrometer. The electron impact ion source was maintained at $200{ }^{\circ} \mathrm{C}$, while the quadrupole temperature was $100^{\circ} \mathrm{C}$. The electron energy was $70 \mathrm{eV}$.

\section{SSCP fingerprint analysis of the rRNA amplicons and sequence determination of fingerprint bands}

The biofilms were harvested with a sterile spatula from three or four PCB droplets and the DNA was extracted using the Fast-DNA-Spin-Kit for soil (Bio 101, CA, USA) following the manufacturer's instructions. The primers chosen for the amplification of bacterial $16 \mathrm{~S}$ rRNA genes were forward primer Com1 and reverse primer Com2-Ph as described by Schwieger \& Tebbe (1998). The phosphorylated strand of the polymerase chain reaction (PCR) products was digested by lambda exonuclease (New England Biolabs, Schwalbach, Germany), and further purified using the Mini-eluteKit (Qiagen, Hilden, Germany) as recommended by the manufacturer, and the remaining single-stranded DNA was dried under vacuum. The DNA was then resuspended in denaturating SSCP loading buffer (47.5\% formamide, $5 \mathrm{mM}$ sodium hydroxide, $0.12 \%(\mathrm{v} / \mathrm{v})$ bromophenol blue, $0.12 \%(\mathrm{v} / \mathrm{v})$ xylene cyanol) and subjected to electrophoresis. Gels were run at $400 \mathrm{~V}$ for $17 \mathrm{~h}$ at $20^{\circ} \mathrm{C}$ in a Macrophor electrophoresis unit (LKB Bromma, Sweden) and subsequently silver stained (Bassam et al., 1991). Single bands were excised from the gels and eluted in extraction buffer $(10 \mathrm{mM}$ Tris-buffer, $5 \mathrm{mM}$ $\mathrm{KCl}, 1.5 \mathrm{mM} \mathrm{MgCl}_{2} .6 \mathrm{H}_{2} \mathrm{O}, 0.1 \%$ Triton X 100, $\mathrm{pH} 9.0$ ) at $95{ }^{\circ} \mathrm{C}$ for $15 \mathrm{~min}$. Extracts were centrifuged (14 000 revolutions per minute, $1 \mathrm{~min}$ ) and the DNA in the supernatant fluid was used for PCR with the primers described above. The PCR product was cleaned (Minielute-Kit (Qiagen)) and sequenced with a sequencing kit (DYEnamic ET Terminator cycle sequencing kit by Amersham Biosciences, Freiburg, Germany) and both primers. The product was cleaned with the Dye Ex Spin Kit (Qiagen) and the sequence analysed on an ABI PRISM ${ }^{\mathrm{TM}} 337$ DNA-Sequencer and a 3100 Genetic Analyser. Comparisons of the sequences were performed using the BLAST program and the databases of EMBL and GenBank.

\section{Phylogenetic analysis}

The phylogenetic analysis (see Fig. 3b) was done using the Clustal_X software (Thompson et al., 1997) for sequence alignments and the neighbour-joining method (Saitou \& Nei, 1987), with bootstrap percentage values based on 1000 replications and the software NJ-Plot (Perrière \& Gouy, 1996), was used to compare sequences and to construct the phylogenetic tree.

\section{Specific polymerase chain reaction for Dehalococcoides}

PCB-dechlorinating microorganisms (Dehalococcoides species) were screened by PCR using specific primers (Watts et al., 2005). Briefly, total DNA from SW-NC1, SW-027 and SW-813 biofilms samples after 45 days of incubation were tested with the primers 14F (5'AGAGTTTGATCCTGGCTCAG-3') and Dehal1265R (5'TATTCCTACCTGCTGTACC-3') in a GeneAmp ${ }^{\circledR}$ PCR System 9700 thermal cycler (Applied Biosystems, USA) using HotStarTaq ${ }^{\circledR}$ DNA Polymerase (Qiagen) with the following cycle parameters: initial denaturation of $15 \mathrm{~min}$ $94^{\circ} \mathrm{C}$, followed by 30 cycles of $30 \mathrm{~s}$ at $94^{\circ} \mathrm{C}$, annealing for $30 \mathrm{~s}$ at $62^{\circ} \mathrm{C}$, elongation for $30 \mathrm{~s}$ at $72^{\circ} \mathrm{C}$ and final extension for $5 \mathrm{~min}$ at $72^{\circ} \mathrm{C}$ (Watts et al., 2005). PCR products were checked on a $1 \%$ TAE (Tris acetate EDTA) agarose gel.

\section{Fluorescence staining and confocal laser scanning microscopy}

The PCB droplet was stained with Nile Red (Sigma, St Louis, MO, USA). Bacterial cell distribution was determined after staining with the nucleic-acid-specific SybrGreen (Molecular Probes Inc., Eugene, OR, USA). 

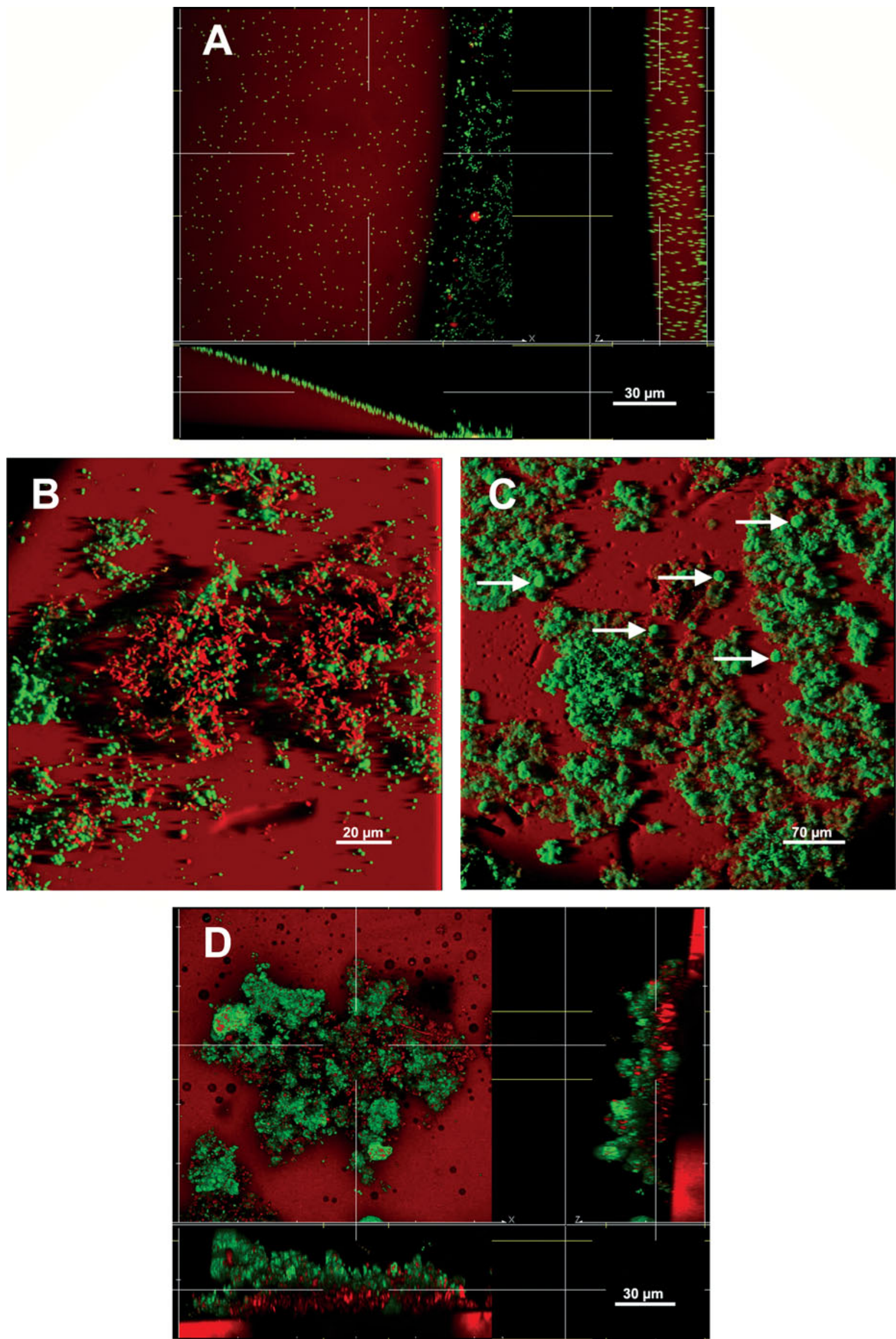

Fig. 2: For legend see facing page. 
The stain was used as supplied and diluted to $5 \mu \mathrm{l}$ in $5 \mathrm{ml}$ of demineralized water. Live and dead cells in biofilms were stained with the BacLight kit (Molecular Probes) and applied as described by the manufacturer. These fluorochromes distinguish living bacteria from compromised bacteria, by means of membrane integrity. The staining solution contained $5 \mathrm{ml}$ of deionized water and $5 \mu \mathrm{l}$ each of the two components SYTO 9 and propidium iodide. This working solution was applied directly to the biofilms. All samples stained in this fashion were examined immediately using epifluorescence and CLSM.

For CLSM, a TCS SP1 attached to an upright microscope was used (Leica, Heidelberg, Germany). From the available laser lines, the line at $488 \mathrm{~nm}$ (SybrGreen) and the $561 \mathrm{~nm}$ (Nile Red) were used for excitation of the fluorochromes. The instrument was controlled by the Leica Confocal software, version 2.61 Build 1537174191. Biofilm samples were observed with $20 \times 0.4 \mathrm{NA}, 63 \times$ 0.9 NA water-immersible lenses. Images were projected by using the microscope software and IMARIS, version 4.06 (Bitplane, Zürich, Switzerland). Images were edited with Photoshop CS (Adobe, San Jose, CA, USA).

\section{Statistical analysis}

For the correlation of metabolic activities and the generation of dendrograms, Statistica 6.0 software (StatSoft, Tulsa, OK, USA) was used. For cluster analysis of PCB transformation, we used the relative amounts of the remaining congeners, the unweighted-pair group method using average linkages, and $1-$ Pearson $r$ as a distance measure.

\section{RESULTS}

\section{Microcosm experiments}

Droplets of $0.7 \mu$ l Aroclor 1242 (NSI Solutions Inc., USA) were placed on sterile Permanox ${ }^{\mathrm{TM}}$ (Nunc, USA) slides $(100 \mathrm{~cm} \times 20 \mathrm{~mm})$. Permanox ${ }^{\mathrm{TM}}$ is a polyester that does not react with PCB. Two slides, each bearing 10 droplets of $\mathrm{PCB}$, were placed in each microcosm, with PCB-droplet side down, on the water surface of a reservoir filled with $500 \mathrm{ml}$ of sterile tap water and $10 \mathrm{~g}$ of the soil sample, shown schematically in Fig. 1b. This resulted in a distance of $6 \mathrm{~cm}$ between PCB droplet and soil sample. Three parallel microcosms were started per soil sample and another three as controls without soil (for PCB analysis), giving 12 microcosms in total. The biofilms were analysed after 45, 75 and 140 days and at each time point one microcosm (two slides) per soil sample, as well as one control, were extracted. After 45 days of incubation, pieces of the Permanox ${ }^{\mathrm{TM}}$ slide with two PCB droplets were cut off and were analysed immediately by CLSM. At the same time point DNA was extracted, and after 45, 75 and 140 days the biofilms were chemically analysed for PCBtransformation as described. After 75 and 140 days DNA extractions from biofilms grown on the PCB oil were not possible because of the strong growth of the biofilm on the Permanox ${ }^{\mathrm{TM}}$ slide in combination with consumption of the PCB droplet, which made it impossible to identify the position of the PCB droplets.

\section{Structure of the biofilms along the pollution gradient}

CLSM was used to examine the structure of PCBgrown biofilms from sites with different levels of PCB contamination. The non-contaminated soil supported a very distinct biofilm type as compared with those from the contaminated sites. The biofilms originating from this soil (Fig. 2a) formed thin bacterial layers that were widespread and rather uniform on the PCB droplet. A slightly denser population of bacteria was found at the margin of the PCB droplet, displaying a higher ratio of bacterial aggregates than did the biofilm on the main area of the PCB droplet (Fig. 2a). In comparison, the biofilms originating from the contaminated soil SW-027 formed agglomerated structures on the PCB droplets (Fig. 2b). To show the position of the PCB droplet, the hydrophobic dye Nile Red was used and, interestingly, this dye stained also some bacterial aggregates, indicating the presence of hydrophobic cell surfaces. These aggregates with hydrophobic surfaces showed no clear preferences for location within the biofilm. Only small differences could be observed between SW-027 and SW-813 biofilms from the PCB-polluted soil samples. One difference was that the biofilms from the heavily contaminated soil SW813 showed a denser coverage of aggregated colonies (biofilm spherules in Fig. 2c). The thickness of the SW813 biofilms reached $50 \mu \mathrm{m}$ and more (Fig. 2d). The biofilms originating from soil sample SW-813 were also treated with Live/Dead stain to determine the ratio of live to damaged cells. The agglomerated structures were all stained green, indicating the presence of living cells. However, it was not possible in this case to decide whether red-stained cells were damaged or merely had hydrophobic surfaces, owing to the possibility of any cells with hydrophobic surfaces being stained simultaneously with Nile Red.

\section{Community analysis: highly diverse biofilm communities}

SSCP community profiling showed highly diverse and distinct microbial communities for each PCB biofilm,

Fig. 2: Confocal laser scanning micrographs of PCB biofilms. (a) Biofilm derived from the non-contaminated soil sample SW-NC1 stained with SybrGreen for DNA (green) and Nile Red for PCB (red); (b) biofilm from soil sample SW-027 stained with SybrGreen and Nile Red. Note here that Nile Red stains also cells; ( $c$ and d) biofilms originating from soil sample SW-813 and treated with Live/Dead stain and Nile Red. The white arrows show the spherical aggregates in this biofilm which are living cells as revealed by their green colour in the Live/Dead staining. 
(a)

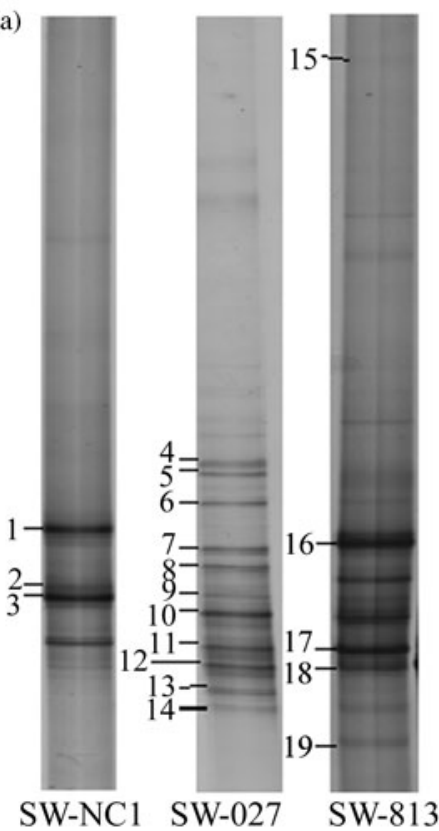

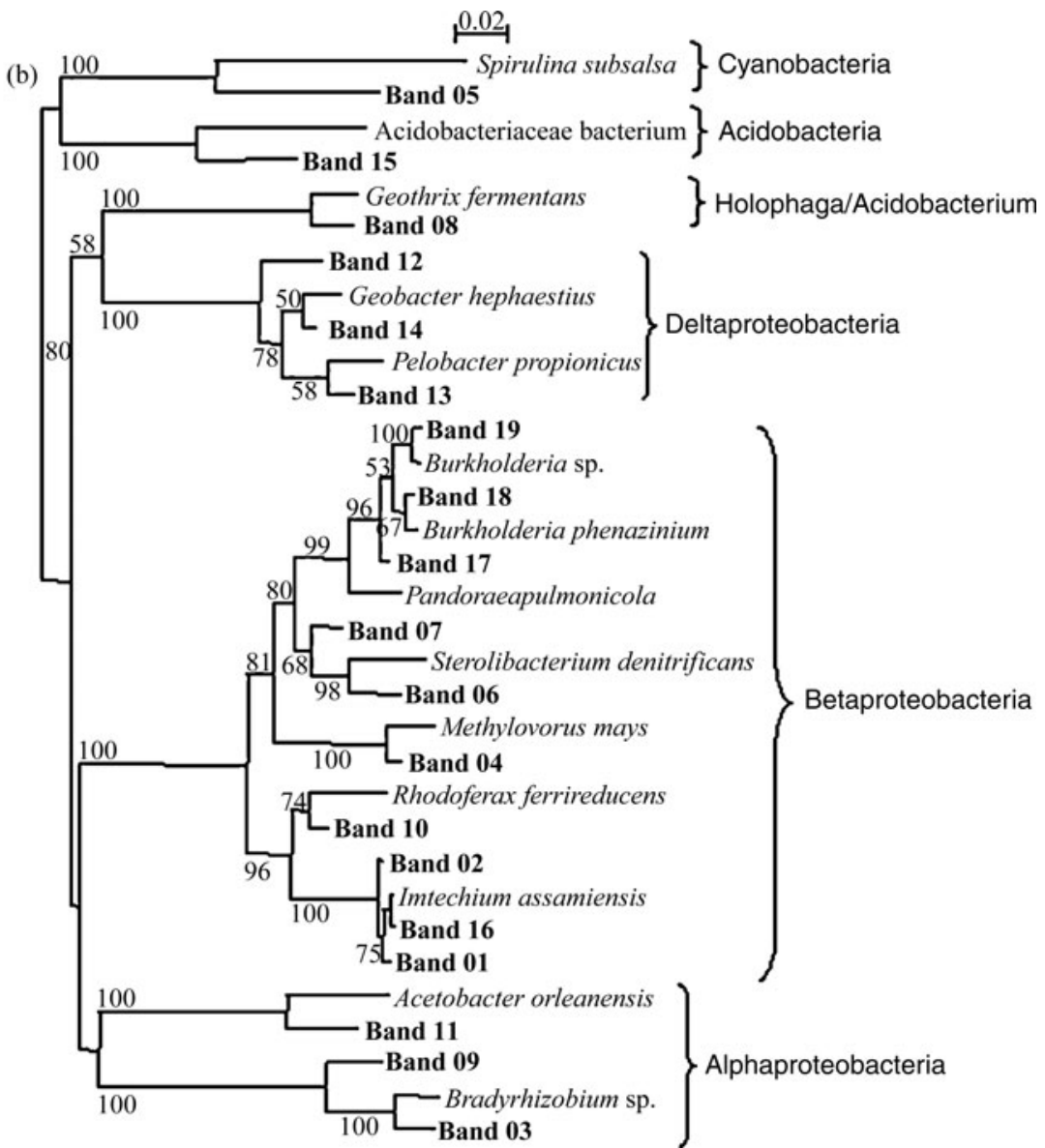

Fig. 3: (a) Composition of PCB biofilm communities obtained from the soil samples SW-NC1, SW- 027 and SW-813 after 45 days of incubation analysed by $16 \mathrm{~S}$ rRNA gene based community fingerprint (SSCP) and (b) phylogeny of their main members.

with the biofilm from the SW-027 soil samples displaying the largest numbers of bands, maybe caused by the slightly higher content of organic carbon in this sample (Fig. 3a). By comparing the sequences of 19 excised bands, 13 different operational taxonomic units (OTUs) could be identified that were closely related: Acetobacter species, Bradyrhizobium species, Burkholderia species, Geobacter species, Geothrix species, Imtechium assamiensis, Methylovorus species, Pandoraea species, Pelobacter species, Rhodoferax species, Spirulina species, Sterolibacterium species as well a species from the Acidobacteriaceae. Of these, only two appeared in more than one biofilm: Bradyrhizobium species and Imtechium assamiensis (in two biofilms). The diversity as well the bacterial relationship is depicted in the phylogenetic tree (Fig. 3b). The majority of the identified OTUs were members of the Betaproteobacteria followed by Deltaproteobacteria and Alphaproteobacteria. Also one OTU respectively from the Cyanobacteria, Acidobacteria and Holophaga/Acidobacterium were detected. A striking difference to the other two biofilms was the dominance of Burkholderia species in biofilms SW-813 (Fig. 3). The search for Dehalococcoides species using the method described by Watts et al. (2005) gave no PCR product in any of the biofilm samples tested.

\section{Transformation of PCB congeners along the pollution gradient}

After 140 days, all biofilms completely transformed the monochlorinated congeners, but biofilms derived from PCB-polluted soil samples achieved this at a significantly faster rate than did the SW-NC1 biofilms. Pronounced differences could be observed in the transformation of PCB congeners between the three biofilms (Table 2) and three groups of congeners could be identified according to their transformations (Fig. 4a). The first group of congeners (Fig. 4a, cluster 1) was transformed by all biofilms but the biofilm communities derived from the contaminated sites did this considerably faster than the biofilms from the non-contaminated soil sample. To this group of congeners belong all mono- and dichlorinated biphenyls, with the exception of 4,4'-dichlorobiphenyl, and 2,4,5-, 2,4,4'/2,4',5- and 2,3',5-trichlorobiphenyl (Table 2). For all these congeners it was observed that the transformation was faster and more complete the higher 
Table 2: PCB transformation (\%) by the biofilms formed on the PCB oil droplet after different time points of incubation

\begin{tabular}{|c|c|c|c|c|c|c|c|c|c|c|}
\hline \multirow{2}{*}{\multicolumn{2}{|c|}{ Incubation time (days) }} & \multicolumn{3}{|c|}{ Biofilm SW-NC1 } & \multicolumn{3}{|c|}{ Biofilm SW-027 } & \multicolumn{3}{|c|}{ Biofilm SW-813 } \\
\hline & & \multirow[t]{2}{*}{45} & \multirow[t]{2}{*}{75} & \multirow[t]{2}{*}{140} & \multirow[t]{2}{*}{45} & \multirow[t]{2}{*}{75} & \multirow[t]{2}{*}{140} & \multirow[t]{2}{*}{45} & \multirow[t]{2}{*}{75} & \multirow[t]{2}{*}{140} \\
\hline $\mathrm{PCB}^{a}$ & $\mathrm{Cl}$ position & & & & & & & & & \\
\hline 1 & 2 & $23 \pm 6^{b}$ & $45 \pm 1$ & 100 & $45 \pm 3$ & $57 \pm 5$ & 100 & $16 \pm 10$ & $77 \pm 14$ & 100 \\
\hline 3 & 4 & - & $32 \pm 1$ & 100 & $5 \pm 0.3$ & $64 \pm 3$ & 100 & $41 \pm 5$ & 100 & 100 \\
\hline 4 & $2,2^{\prime}$ & $5 \pm 0.2$ & $20 \pm 0.2$ & $57 \pm 8$ & $5 \pm 0.2$ & $27 \pm 0.8$ & $73 \pm 10$ & $<5 \pm 0.2$ & $35 \pm 6$ & $66 \pm 4$ \\
\hline $5 / 8$ & $2,3 / 2,4^{\prime}$ & - & $10 \pm 0.2$ & $79 \pm 23$ & $<5 \pm 0.1$ & $27 \pm 1$ & $89 \pm 43$ & $5 \pm 0.2$ & $90 \pm 7$ & 100 \\
\hline 6 & $2,3^{\prime}$ & - & $11 \pm 0.3$ & $62 \pm 4$ & $<5 \pm 0.03$ & $16 \pm 0.2$ & $67 \pm 15$ & $19 \pm 0.2$ & $61 \pm 19$ & $93 \pm 9$ \\
\hline $7 / 9$ & $2,4 / 2,5$ & $6 \pm 0.4$ & $20 \pm 0.1$ & $66 \pm 7$ & $8 \pm 0.2$ & $26 \pm 0.6$ & $71 \pm 20$ & $19 \pm 1$ & $62 \pm 15$ & $93 \pm 7$ \\
\hline 13 & $3,4^{\prime}$ & - & - & $59 \pm 9$ & $<5 \pm 0.2$ & $7 \pm 0.8$ & $57 \pm 6$ & $7 \pm 0.1$ & $35 \pm 13$ & $71 \pm 8$ \\
\hline $15 / 17$ & $4,4^{\prime} / 2,2^{\prime}, 4$ & - & - & $24 \pm 2$ & - & $6 \pm 0.05$ & $31 \pm 4$ & - & $<5 \pm 0.04$ & $5 \pm 0.1$ \\
\hline $16 / 32$ & $2,2^{\prime}, 3 / 2,4^{\prime}, 6$ & - & $<5 \pm 0.04$ & $14 \pm 0.6$ & - & $<5 \pm 0.04$ & $22 \pm 2$ & - & - & - \\
\hline 18 & $2,2^{\prime}, 5$ & - & $<5 \pm 0.01$ & $16 \pm 1$ & - & $<5 \pm 0.03$ & $26 \pm 2$ & - & $<5 \pm 0.04$ & $<5 \pm 0.8$ \\
\hline 19 & $2,2^{\prime}, 6$ & - & $<5 \pm 0.03$ & $18 \pm 0.2$ & - & $5 \pm 0.1$ & $32 \pm 1$ & - & $<5 \pm 0.02$ & $<5 \pm 0.03$ \\
\hline 26 & $2,3^{\prime}, 5$ & - & - & $15 \pm 0.6$ & - & $<5 \pm 0.02$ & $29 \pm 4$ & - & $9 \pm 0.9$ & $51 \pm 2$ \\
\hline 27 & $2,3^{\prime}, 6$ & - & $7 \pm 0.1$ & $24 \pm 0.4$ & - & $9 \pm 0.08$ & $17 \pm 0.7$ & - & - & - \\
\hline $28 / 31$ & $2,4,4^{\prime} / 2,4^{\prime}, 5$ & $<5 \pm 0.04$ & $<5 \pm 0.01$ & $20 \pm 2$ & $<5 \pm 0.05$ & $5 \pm 0.06$ & $41 \pm 5$ & $16 \pm 0.4$ & $23 \pm 3$ & $81 \pm 27$ \\
\hline 29 & $2,4,5$ & - & - & $8 \pm 0.4$ & - & $<5 \pm 0.03$ & $12 \pm 1$ & $5 \pm 0.1$ & $12 \pm 0.6$ & $44 \pm 6$ \\
\hline 34 & $2^{\prime}, 3,5$ & $<5 \pm 0.01$ & $<5 \pm 0.1$ & $9 \pm 0.4$ & - & $<5 \pm 0.02$ & $12 \pm 0.2$ & - & $<5 \pm 0.05$ & $6 \pm 0.2$ \\
\hline $56 / 60$ & $2,3,3^{\prime}, 4^{\prime} / 2,3,4,4^{\prime}$ & - & - & - & - & - & - & $<5 \pm 0.4$ & $34 \pm 20$ & $40 \pm 15$ \\
\hline $66 / 80$ & $2,3^{\prime}, 4,4^{\prime} / 3,3^{\prime}, 5,5^{\prime}$ & - & - & - & - & - & - & $5 \pm 0.4$ & $26 \pm 10$ & $33 \pm 14$ \\
\hline 87 & $2,2^{\prime}, 3,4,5^{\prime}$ & - & - & - & - & - & - & $5 \pm 0.4$ & $20 \pm 7$ & $34 \pm 9$ \\
\hline 110 & $2,3,3^{\prime}, 4^{\prime}, 6$ & - & - & - & - & - & - & $<5 \pm 1$ & $23 \pm 4$ & $39 \pm 11$ \\
\hline
\end{tabular}

${ }^{a}$ Identified by IUPAC number.

${ }^{b}$ Standard deviation based on three measurements.

the PCB contamination was in the soil inoculum. The second group (cluster 2) of congeners comprised more highly chlorinated biphenyls that were only transformed by the SW-813 biofilms. In this group of congeners fell all tetra- and pentachlorinated congeners $\left(2,2^{\prime}, 3,4,5^{\prime}\right.$ -

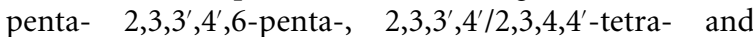
$2,3^{\prime}, 4,4^{\prime} / 3,3^{\prime}, 5,5^{\prime}$-tetrachlorobiphenyl). Soil samples from the medium-polluted and non-polluted sites failed to generate biofilms able to transform these PCBs. Finally, a third group (cluster 3 ) of congeners could be identified that were transformed by SW-NC1 and SW-027 biofilms but apparently not by SW-813 biofilms. Into this group fell 2,3',6-, 2',3,5-, 2,2',6-, 2,2',5-tri-, 4,4'-di-/2,2',4-tri-, and 2,2',3-/2,4',6-trichlorobiphenyl. This comprises all the trichlorinated congeners found in Aroclor 1242 with the exception of the $2,4,4^{\prime}-12,4^{\prime}, 5$-trichlorobiphenyl, which belonged to the first group.

The transformation of the individual congeners of the biofilms at different time points was used to group the metabolic activities of the biofilms. Fig. $4 \mathrm{~b}$ reveals that the activities of biofilms from the non-contaminated and the slightly contaminated soil samples were rather similar and that time was a much more important factor for PCB transformation activity than the biofilm communities developing from the soil samples because NC-1 and SW027 biofilm samples from the same time points clustered together. However, this observation was not valid for the biofilms from the highly contaminated soil sample. Here, from the very beginning, the PCB transformation activity of the biofilm community was different from the other biofilms, although a considerable change in PCB transformation over time was seen as well.

\section{DISCUSSION}

Clear differences in the structure of the biofilms, in the composition of the biofilm communities and in their ability to transform the different PCB congeners were observed along the pollution gradient. The PCB biofilms showed either homogeneous, thin, single-cell coverage or aggregated biofilms, some of which contained spherical microcolonies. This observation is in agreement with reports on the colonization of diesel droplets by Acinetobacter (Baldi et al., 1999). However, in the study by Baldo et al. the diesel droplets were broken down to microdroplets owing to the production of surface active compounds. Whyte et al. (1999) studied the colonization of diesel droplets by a Rhodococcus strain and demonstrated an even coverage of the droplets by the rodshaped bacteria. Furthermore they could demonstrate the colonization of liquid and solid hydrocarbon substrates. This observation is probably related to the PCB biofilms, although Gram-positive bacteria were not detected in these microbial communities.

PCB transformation was very distinct between the biofilms, with a surprisingly high activity displayed by the non-adapted community from site SW-NC1. SW813 biofilms showed the highest performance in PCB transformation, in the degree of transformation, the 

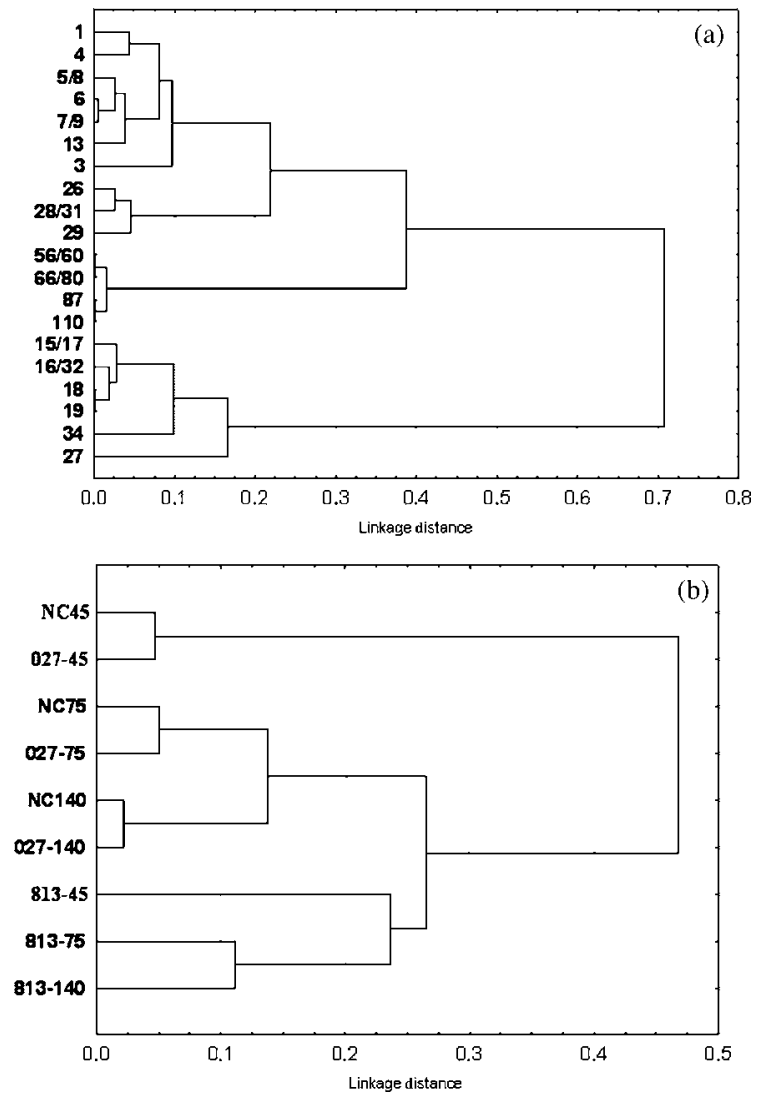

Fig. 4: (a) Dendrogram of PCB congeners resulting from a cluster analysis based on their transformation by the different biofilm communities at different time points. Individual congeners are identified by their IUPAC number (see Table 2). (b) Dendrogram of the PCB transformation activities of the biofilms at different time points derived from a cluster analysis based on the transformation of PCB congeners. The biofilm communities are named after their soil sample inocula and the time point of sampling; for example, $813-140$ is the biofilm derived from soil sample SW-813 after 140 days. Both dendrograms were based on Pearson coefficient and unweighted pair group method with arithmetic mean (UPGMA).

diversity of congeners attacked and the transformation rates, followed by the SW-027 biofilms from the medium-contaminated site and then by the SW-NC1 biofilms. The most significant differences were the transformation of tetra- and pentachlorobiphenyls only by biofilm SW-813 and the very slow transformation of trichlorobiphenyls by this biofilm community, the latter being in many cases even slower than in SW$\mathrm{NC1}$ biofilms. One explanation for this phenomenon could be the much lower activities in trichlorobiphenyl transformation of the SW-813 biofilms. An alternative scenario would be a transformation of this group of congeners comparable to the transformation by other biofilm communities but disguised by the replenishment of trichlorobiphenyls formed by anaerobic dechlorination of pentachlorobiphenyl congeners. Although the SW-813 biofilm transformed trichlorobiphenyls to a very small extent, it showed a pronounced transformation of the pentachlorinated congeners and it seems reasonable to explain these two facts by anaerobic transformation.
Reductive dechlorination of PCB congeners was reported to be preferentially a meta-reduction with little parareduction (Wiegel \& Wu, 2000). For example, it has been observed that 2,3,3',4',6-pentachlorobiphenyl (PCB 110) is reductively dechlorinated to $2,3^{\prime}, 4^{\prime}, 6-$ tetrachlorobiphenyl, which itself is reduced to $2,4^{\prime}, 6-(\mathrm{Wu}$ et al., 1998) or 2,3',6-trichlorobiphenyl, congeners apparently not transformed by this biofilm community. The reductive chlorination of 2,2',3,4,5-pentachlorobiphenyl to 2,2',4- and 2,2',5-trichlorobiphenyl (Wiegel \& Wu, 2000; Nollet et al., 2005), 2,3,4,4'-tetrachlorobiphenyl or $2,3^{\prime}, 4,4^{\prime}$-tetrachlorobiphenyl to 2,4,4'-trichlorobiphenyl (Baldi et al., 1999) and 2,3,3',4'-tetrachlorobiphenyl to $2,4^{\prime}$-dichlorobiphenyl readily undergone by this biofilm could explain most of the apparent nontransformation of trichlorobiphenyls by the SW-813 biofilms.

Reductive dehalogenation requires anaerobic conditions and an electron donor within a biofilm that simultaneously transforms low chlorinated biphenyls by aerobic dioxygenation. To achieve these two different transformation regimens, anaerobic pockets within the biofilm are needed. One of the main differences between the biofilm derived from the non-contaminated soil sample and biofilms originating from PCB-polluted soil is the formation of aggregates within the biofilm. The formation of these aggregates was very prominent in SW-813 biofilms, as shown in the three-dimensional structure (see Fig. 2d). The PCB biofilm had an aerobic site at the water-biofilm interface and an oxygen depleted site at the biofilm-substratum interface. We speculate that the aggregates in this biofilm community reached a critical size that would limit the oxygen supply in their interior, allowing reductive dechlorination. Borriello et al. (2004) have shown that, in a biofilm of Pseudomonas aeruginosa, only the upper $30 \mu \mathrm{m}$ could support aerobic metabolism. This is about the size of some of the biofilm aggregates, although the SW-813 bofilm achieved up to $50 \mu \mathrm{m}$ thickness. In a complex microbial community comprising many bacterial species not known to degrade PCB and probably having unknown functions in the biofilm, the electron acceptors in the presumed anaerobic dechlorination remain unknown (Holliger et al., 1999). Such a scenario would also allow a transport by diffusion of the end-products of the reductive chlorination (medium-chlorinated congeners) to the surface of the aggregates, where they would be aerobically metabolized. The absence of Dehalococcoides species in the biofilms, determined with specific primer, does not necessarily contradict our hypothesis of anaerobic dechlorination in the biofilm pockets, since a number of other strains able to dehalogenate chlorinated compounds have been described (El Fantroussi et al., 1998). Even for hydroxylated polychlorinated biphenyls structurally similar to PCB Desulfitobacterium dehalogenans was described as being a reductive dechlorinator (Wiegel et al., 1999).

Comparative community analysis of the three biofilm communities did not allow the identification of the taxa performing the reductive dechlorination. The 19 
identified SSCP bands showed 13 distinct species and the biofilm from the medium-contaminated soil SW027 possessed the highest diversity. Interestingly, although sample collection was done within a very small area, each soil sample collected gave a considerably different biofilm community on PCB oil. Contrary to the other biofilm communities, the identified species of SW-813 biofilms showed a high abundance of Burkholderia species, already described as some of the most active species at this site (Nogales et al., 1999) and shown to be the main degraders of PCB in biofilm communities growing on PCB oil (Tillmann et al., 2005). Burkholderia species, however, are not always prominent members of biofilm communities growing on PCB oil using soil samples from this site as inocula, as has been reported before (Macedo et al., 2005) and above in the community analyses of SW-NC1 and SW-027 biofilms.

The results of our study revealed diverse microbial communities in the pristine soil that were able to use the lower chlorinated congeners of the xenobiotic PCB. A high degree of PCB pollution in the soil correlated with high biotransformation activity of PCB of the microbial communities. We can only speculate how the microbial communities adapted to the hydrophobic PCB. Optimization of PCB-consuming consortia by gene transfer in the last decade, as has been reported for the utilization of chlorobenzoate (Focht et al., 1996; Top et al., 2002), may have played a role either by means of an exchange of microorganisms between the three closely spaced sampling sites or by other community conditioning (Langworthy et al., 1998; Dahllof et al., 2001). One important result of these optimizations is the improved formation of aggregates in the biofilms thick enough to allow the exclusion of oxygen from their interior and the possible onset of reductive dechlorination. We hypothesize that this aggregate formation enables the microbial community of the PCB-polluted sites to use more highly chlorinated congeners, which are first reduced to medium-chlorinated compounds and then aerobically metabolized. Our results present a glimpse of the complex interactions between microorganisms in polluted environments and offer first insights into how the metabolic potential of microbial communities shift with increasing PCB content in soil.

\section{ACKNOWLEDGEMENTS}

We are indebted to Jennifer Skerra for microbiological work and Esther Surges for PCB and soil analysis. A.J.M. acknowledges the support by a Ph.D. stipend from the German Academic Exchange Service (DAAD).

\section{REFERENCES}

Abraham, W.-R., Nogales, B., Golyshin, P. N., Pieper, D. H. \& Timmis, K. N. (2002) Polychlorinated biphenyl-degrading microbial communities in soils and sediments. Current Opinion in Microbiology 5, 246-253

Ayris, S. \& Harrad, S. (1999) The fate and persistence of polychlorinated biphenyls in soil. Journal of Environmental Monitoring 1, 395-401

Baldi, F., Ivošević, N., Minacci, A., Pepi, M., Fani, R., Svetličić, V. \& Žutić, V. (1999) Adhesion of Acinetobacter venetianus to diesel fuel droplets studied with in situ electrochemical and molecular probes. Applied and Environmental Microbiology 65, 2041-2048

Ballschmiter, K. \& Zell, M. (1980) Baseline studies of the global pollution. I. Occurrence of organohalogens in pristine European and Antarctic aquatic environments. International Journal of Environmental Analytical Chemistry 8, 15-35

Bassam, B. J., Caetano-Anolles, G. \& Gresshoff, P. M. (1991) Fast and sensitive silver staining of DNA in polyacrylamide gels. Analytical Biochemistry 196, 80-83

Borriello, G., Werner, E., Roe, F., Kim, A. M., Ehrlich, G. D. \& Stewart, P. S. (2004) Oxygen limitation contributes to antibiotic tolerance of Pseudomonas aeruginosa in biofilms. Antimicrobial Agents and Chemotherapy 48, 2659-2664

Buthe, A. \& Denker, E. (1995) Qualitative and quantitative determination of PCB congeners by using a HT-5 GC column and an efficient quadropole MS. Chemosphere 30, 753-771

Dahllof, I., Agrenius, S., Blanck, H., Hall, P., Magnusson, K. \& Molander, S. (2001) The effect of TBT on the structure of a marine sediment community - a boxcosm study. Marine Pollution Bulletin 42, 689-695

Duinker, J. C., Schulz, D. E. \& Petrick, G. (1988) Selection of chlorinated biphenyl congeners for analysis in environmental samples. Marine Pollution Bulletin 19, 19-25

El Fantroussi, S., Naveau, H. \& Agathos, S. N. (1998) Anaerobic dechlorinating bacteria. Biotechnology Progress 14, 167-188

Focht, D. D., Searles, D. B. \& Koh, S. C. (1996) Genetic exchange in soil between introduced chlorobenzoate degraders and indigenous biphenyl degraders. Applied and Enviromental Microbiology 62, 3910-3913

Hall-Stoodley, L., Costerton, J. W. \& Stoodley, P. (2004) Bacteria biofilms: from the natural environment to infectious diseases. Nature Reviews Microbiology 2, 95-108

Harkness, M. R., McDermott, J. B., Abramowicz, D. A., Salvo, J. J., Flanagan, W. P., Stephens, M. L., et al. (1993) In situ stimulation of aerobic PCB biodegradation in Hudson River sediments. Science 259, 503-507

Holliger, C., Wohlfarth, G. \& Diekert, G. (1999) Reductive dechlorination in the energy metabolism of anaerobic bacteria. FEMS Microbiology Reviews 22, 383-398

Ivanov, V. \& Sandell, E. (1992) Characterization of polychlorinated biphenyl isomers in Sovol and trichlorobiphenyl formulations by high-resolution gas chromatography with electron capture detection and high-resolution gas chromatography-mass spectrometry techniques. Environmental Science \& Technology 26, 2012-2017

Jensen, S., Johnels, A. G., Olsson, M. \& Otterlind, G. (1969) DDT and PCB in marine animals from Swedish waters. Nature 224, $247-250$.

Langworthy, D. E., Stapleton, R. D., Sayler, G. S. \& Findlay, R. H. (1998) Genotypic and phenotypic responses of a riverine microbial community to polycyclic aromatic hydrocarbon contamination. Applied and Environmental Microbiology 64, $3422-3428$

Lünsdorf, H., Erb, R.W., Abraham, W.-R. \& Timmis, K. N. (2000) "Clay hutches": a novel interaction between bacteria and clay minerals. Environmental Microbiology 2, 161-168

Macedo, A. J., Kuhlicke, U., Neu, T. R., Timmis, K. N. \& Abraham, W.-R. (2005) Three stages of a biofilm community developing at the liquid-liquid interface between polychlorinated biphenyls and water. Applied and Environmental Microbiology 71, 7301-7309

Nogales, B., Moore, E. R. B., Abraham, W.-R. \& Timmis, K. N. 
(1999) Identification of the metabolically active members of a bacterial community in a polychlorinated biphenyl polluted moorland soil. Environmental Microbiology 1, 199-212

Nogales, B., Moore, E. R. B., Llobet-Brossa, E., Rossello-Mora, R., Amann, R. \& Timmis, K. N. (2001) Combined use of $16 \mathrm{~S}$ ribosomal DNA and 16S rRNA to study the bacterial community of polychlorinated biphenyl-polluted soil. Applied and Environmental Microbiology 67, 1874-1884

Nollet, H., Van de Putte, I., Raskin, L. \& Verstraete, W. (2005) Carbon/electron source dependence of polychlorinated biphenyl dechlorination pathways for anaerobic granules. Chemosphere 58, 299-310

Perrière, G. \& Gouy, M. (1996) WWW-Query: an on-line retrieval system for biological sequence banks. Biochimie 78, 364-369

Ross, G. (2004) The public health implications of polychlorinated biphenyls (PCBs) in the environment. Ecotoxicology and Environmental Safety 59, 275-291

Saitou, N. \& Nei, M. (1987) The neighbor-joining method: a new method for reconstructing phylogenetic trees. Molecular Biology and Evolution 4, 406-425

Schachter, B. (2003) Slimy business - the biotechnology of biofilms. Nature Biotechnology 21, 361-365

Schwieger, F. \& Tebbe, C. C. (1998) A new approach to utilize PCR-single-strand-conformation polymorphism for 16S rRNA gene-based microbial community analysis. Applied and Environmental Microbiology 64, 4870-4876

Thompson, J. D., Gibson, T. J., Plewniak, F., Jeanmougin, F. \& Higgins, D. G. (1997) The CLUSTAL_X windows interface: flexible strategies for multiple sequence alignment aided by quality analysis tools. Nucleic Acids Research 25, 48764882
Tillmann, S., Strömpl, C., Timmis, K. N. \& Abraham, W.-R. (2005) Stable isotope probing reveals the dominant role of Burkholderia species in aerobic degradation of PCBs. FEMS Microbiology Ecology 52, 207-217

Top, E. M., Springael, D. \& Boon, N. (2002) Catabolic mobile genetic elements and their potential use in bioaugmentation of polluted soils and waters. FEMS Microbiology Ecology 42, 199-208

Walker, J. T., Bradshaw, D. J., Fulford, M. R. \& Marsh, P. D. (2003) Microbiological evaluation of a range of disinfectant products to control mixed-species biofilm contamination in a laboratory model of a dental unit water system. Applied and Environmental Microbiology 69, 3327-3332

Watts, J. E., Fagervold, S. K., May, H. D. \& Sowers, K. R. (2005) A PCR-based specific assay reveals a population of bacteria within the Chloroflexi associated with the reductive dehalogenation of polychlorinated biphenyls. Microbiology 151, 2039-2046

Whyte, L. G., Slagman, S. J., Pietrantonio, F., Bourbonnière, L., Koval, S. F., Lawrence, J. R., et al. (1999) Physiological adaptations involved in alkane assimilation at a low temperature by Rhodococcus sp. strain Q15. Applied and Environmental Microbiology 65, 2961-2968

Wiegel, J. \& Wu, Q. (2000) Microbial reductive dehalogenation of polychlorinated biphenyls. FEMS Microbiology Ecology 32, 1-15

Wiegel, J., Zhang, X. \& Wu, Q. (1999) Anaerobic dehalogenation of hydroxylated polychlorinated biphenyls by Desulfitobacterium dehalogenans. Applied and Environmental Microbiology 65, 2217-2221

Wu, Q., Sowers, K. R \& May, H. D. (1998) Microbial reductive dechlorination of Aroclor 1260 in anaerobic slurries of estuarine sediments. Applied and Environmental Microbiology 64, 1052-1058 